\title{
Experiência de pacientes com acupuntura no Sistema Único de Saúde em diferentes ambientes de cuidado e (des)medicalização social
}

\author{
Acupuncture patients' experience in the \\ Brazilian Unified National Health System \\ in different healthcare settings and social \\ (de)medicalization
}

\section{La experiencia de los pacientes con tratamiento de acupuntura en el Sistema de Salud de Brasil en diferentes ámbitos de atención y la (des)medicalización social}

Emiliana Domingues Cunha da Silva 1 Charles Dalcanale Tesser 2

\footnotetext{
1 Programa de Pósgraduação em Saúde Coletiva, Universidade Federal de Santa Catarina, Florianópolis, Brasil.

2 Departamento de Saúde Pública, Universidade Federal de Santa Catarina, Florianópolis, Brasil.

Correspondência E. D. C. Silva Programa de Pós-graduação em Saúde Coletiva, Universidade Federal de Santa Catarina.

Rua Amaro Antonio Vieira 1200, bloco 1, apto. 301, Florianópolis, SC 88034-100, Brasil. emidomingues@hotmail.com
}

\begin{abstract}
The virtues attributed to complementary therapies such as holistic and patient-centered approaches and stimulus for self-healing have been increasingly valued and could theoretically attenuate the current prevailing excessive social medicalization. Among such therapies, acupuncture has been highlighted due to its progressive institutionalization and acceptance. The objective of the current study was to analyze the experience of acupuncture patients in the Brazilian Unified National Health System and its relationship to their care, in light of social medicalization in terms of the constitutive aspects, representations, and users' explanatory models. Thirty patients were interviewed, selected from primary and secondary care services in Florianópolis, Santa Catarina State, Brazil. Important therapeutic results were observed in the principal complaints, sleep, disposition, emotional status, and decreased use of medication. Users' explanatory models and self-care were modified very little, or not at all, by acupuncture treatment. However, the study showed expanded and less iatrogenic care, especially in the primary care setting, in which there was little supply of other associated practices from Chinese medicine.
\end{abstract}

Acupunture; Complementary Therapies; Self Care; Primary Health Care

\section{Resumo}

Virtudes atribuídas às terapias complementares, como abordagem holística e centrada nos pacientes e estímulo à autocura, vêm sendo valorizadas e podem, em tese, atenuar a excessiva medicalização social hoje vigente. Dentre elas, a acupuntura destaca-se pela institucionalização e aceitação progressiva. O objetivo foi analisar a experiência de pacientes com acupuntura no Sistema Único de Saúde e como ela se insere no seu cuidado, à luz da medicalização social, esta pensada nos seus aspectos constitutivos das representações e modelos explicativos dos usuários. Foram entrevistados trinta pacientes selecionados das atenções primária e secundária de Florianópolis, Santa Catarina, Brasil. Observou-se relevantes resultados terapêuticos nas queixas principais, no sono, na disposição, no estado emocional e diminuição do uso de fármacos. Os modelos explicativos dos usuários e seu autocuidado foram pouco ou nada modificados pelo tratamento com acupuntura. Notou-se, todavia, um cuidado ampliado e menos iatrogênico, principalmente na atenção primária à saúde, em que houve pouca oferta de outras práticas da medicina chinesa associadas.

Acupuntura; Terapias Complementares; Autocuidado; Atenção Primária à Saúde 


\section{Introdução}

A Organização Mundial da Saúde (OMS) 1 tem reconhecido a importância das chamadas medicinas tradicionais e medicinas alternativas e complementares (MAC) em todo o mundo. Dentre elas, destaca-se a medicina tradicional chinesa (MTC), que se configura como uma racionalidade médica. A categoria "racionalidade médica" foi criada por Luz ${ }^{2}$ para estudar sistemas médicos complexos. Permite compreendê-los e analisá-los sem tomar como critério de verdade a biomedicina, que passa a ser mais um sistema de cura a ser compreendido, dentre outros. A MTC engloba uma série de práticas terapêuticas além da acupuntura, objeto desta pesquisa, como fitoterapia, massagens, práticas corporais, dieta etc.

A acupuntura vem ganhando popularidade e aceitação no ocidente 3 , sendo fomentada no Brasil pela Política Nacional de Práticas Integrativas e Complementares (PNPIC) 4,5. A PNPIC incentiva a inserção dessas práticas no Sistema Único de Saúde (SUS) em todos os níveis de atenção, com ênfase na atenção primária.

Poucos estudos tematizaram a atuação da MTC/acupuntura no SUS. Freire Jr. ${ }^{6}$ registrou uma experiência brasileira de uso de modalidades da MTC voltado para a educação em saúde e o autocuidado. Cintra \& Figueiredo 7 observaram que o contato com a acupuntura incentiva uma postura ativa e a visão crítica dos pacientes sobre seu próprio corpo, propiciando melhor autocuidado. Neste estudo e em outro na Espanha 8, os pacientes relataram que a acupuntura reduz custos e consumo de medicamentos alopáticos. Isso faz pensar que ela é relevante, principalmente frente à iatrogenia significativa do cuidado médico, que está cada vez mais em evidência com o atual processo de medicalização social. Este consiste na transformação progressiva de vivências e sofrimentos antes administrados pelas pessoas autonomamente em problemas médicos. Está relacionado à expansão tecnológica e social da biomedicina, à mercantilização dos cuidados, à propaganda midiática de tecnologias biomédicas e à expansão do chamado complexo médicoindustrial 9,10.

A medicalização interfere culturalmente nas populações, com um declínio da capacidade de enfrentamento autônomo de parte dos adoecimentos. O processo tende a penetrar em todos os setores da vida. Ele não é em si necessariamente negativo ou positivo ${ }^{9}$. Todavia, no contexto do cuidado profissional no SUS, há certa identificação do termo "medicalização" com o seu excesso, sustentada pela frequente medicalização abusiva de vivências e aspectos da vida, associada a uma comum redução e restrição dos significados e dos cuidados (autônomos e heterônomos) às categorias nosológicas biomédicas e aos seus tratamentos consagrados (quimioterapia e cirurgia).

Outro aspecto da medicalização, mais sutil, é a geração de dependência excessiva em situações de franco adoecimento sentido, com redução dos cuidados ao consumo de serviços e produtos e ao cumprimento das prescrições profissionais, com acentuada desconsideração de fatores sociais, emocionais, ambientais, subjetivos, morais e culturais. $\mathrm{O}$ saber biomédico carrega um reducionismo biológico 11 , que é um potente motor epistemológico e cultural da medicalização social. Isso é particularmente relevante nos adoecimentos crônicos e acentuado com o envelhecimento populacional, situações em que há, por um lado, frequentemente, limitação explicativa/interpretativa e de eficácia terapêutica e, por outro, grande potencial de iatrogenia clínica na biomedicina 12 . Nessas situações, os desafios são grandes em termos de necessidades e dificuldades de "empoderamento" - psicológico e comunitário, conforme Carvalho \& Gastaldo 13 - e de fomento à maior autonomia, autocuidado, ressignificação das experiências e adaptação dos usuários aos adoecimentos, tratamentos e limitações a eles associados. É sobre esse aspecto da medicalização que incidiu um dos interesses e focos desta pesquisa. Há defensores de que a pluralidade dos cuidados em saúde no SUS, por meio da oferta de práticas e MAC, poderia ter um efeito de contenção ou desvio da medicalização excessiva 14,15. A acupuntura e a MTC seriam uma possibilidade relevante e viável nesse sentido 7,16,17,18,19. A hipótese subjacente é que a maior integralidade na abordagem, as várias teorias sobre saúde/doença, a distinta concepção de saúde vitalista da MTC e de outras MAC e racionalidades médicas, e suas diversas modalidades de cuidado (heterônomas e autônomas), são potenciais recursos desmedicalizantes, "empoderadores" e enriquecedores das experiências de cuidado no SUS.

Por outro lado, a acupuntura é um tipo de cuidado heterônomo dos mais similares e assimiláveis à cultura medicalizada, ao imaginário e aos procedimentos biomédicos e, não por acaso, talvez seja uma das técnicas mais aceitas e institucionalizadas das MAC, que vêm se expandindo aos poucos no SUS 4 . Nesse contexto, ela corre o risco de padecer dos males da (hiper)medicalização. Essa situação a torna um relevante objeto de estudo, enfocando como sua prática tem interagido com os doentes, e quanto das virtudes a ela atribuídas (abordagem mais holística, centramento do cuidado na pessoa, estímulo ao potencial de autocura etc.) estão presentes, como 
atuam e como são percebidas nas experiências dos usuários.

O objetivo desta pesquisa foi investigar a experiência de usuários de acupuntura do SUS de Florianópolis, Santa Catarina, Brasil, sobre seu tratamento, incluindo sua percepção sobre eficácia, redução do uso de medicamentos, orientações recebidas, mudanças no autocuidado e nos modelos explicativos dos usuários, do ponto de vista da medicalização no foco supramencionado. Outro objetivo foi investigar essa experiência em diferentes ambientes de cuidado - atenção primária à saúde e atenção secundária, testando, de modo exploratório, a hipótese de que a atenção primária à saúde pode, por hipótese, ser um ambiente mais favorável à racionalidade da MTC e à acupuntura, pelo contexto de fácil acesso, longitudinalidade, maior intimidade da relação de cura, maior conhecimento dos usuários, maior abertura para outras formas de cuidado e, portanto, condições mais favoráveis a um cuidado menos medicalizante.

\section{Método}

Trata-se de um estudo descritivo com abordagem qualitativa, envolvendo usuários de acupuntura no SUS. A coleta de dados foi realizada entre outubro de 2011 e abril de 2012, em Florianópolis, onde as práticas de acupuntura são realizadas na atenção primária à saúde e na atenção secundária (policlínicas municipais e hospital universitário da Universidade Federal de Santa Catarina UFSC). Havia um médico acupunturista em cada uma das duas policlínicas e cerca de vinte profissionais da Estratégia Saúde da Família (ESF) com especialização em acupuntura, mas poucos a exerciam. Os atendimentos nas policlínicas e no ambulatório do hospital universitário tinham uma lista de espera de pacientes encaminhados pelos médicos do município, ao contrário dos entrevistados na atenção primária à saúde que eram selecionados pelos próprios médicos da ESF (também acupunturistas).

Realizaram-se entrevistas semiestruturadas, com usuários selecionados a partir da sexta sessão de tratamento (pelo fato de estes estarem mais familiarizados com a terapêutica e disporem de uma mínima experiência com ela a ser investigada), acima de 18 anos, atendidos na atenção primária à saúde e na atenção secundária, escolhidos aleatoriamente, seguindo o critério de saturação dos dados 20,21. No total, foram trinta entrevistados, sendo dez nas duas policlínicas, sete no hospital universitário e treze em três centros de saúde. As entrevistas foram realizadas nos serviços de saúde logo após os atendimentos, e duraram em média cerca de trinta minutos (variando de 20 a 45 minutos). Seguiram-se todos os procedimentos previstos na Resolução no 196/96 do Conselho Nacional de Saúde quanto aos cuidados éticos e aprovação no Comitê de Ética em Pesquisa da UFSC (no 2124/12).

As entrevistas foram gravadas, transcritas e pré-analisadas conforme Bardin 22, com leitura e releitura a fim de identificar a percepção dos sujeitos sobre o tratamento. Em seguida, foram detectadas as "unidades de significado", cujos temas recorrentes foram agrupados. A análise foi em parte dirigida por categorias prévias relacionadas a interesses da pesquisa e em parte aberta a temas emergentes, na perspectiva de uma compreensão do fenômeno do ponto de vista do entrevistado e de um olhar crítico ancorado no tema da medicalização social.

\section{Resultados e discussão}

Grande parte dos entrevistados era do sexo feminino, como regra em serviços ambulatoriais em geral e em perfis de pacientes usuários de acupuntura no ocidente 7 . A maioria era de meia idade, entre 30 e 60 anos, e com Ensino Fundamental (Tabela 1).

\section{Situações e motivos para a chegada à acupuntura}

Apesar de a acupuntura pertencer a uma medicina milenar, permanece ainda pouco conhecida por muitos profissionais de saúde, que a utilizam quando as outras intervenções não apresentam resultado. Isso se reflete no encaminhamento aos acupunturistas da atenção secundária: " $O$ médico me recomendou acupuntura porque os outros tratamentos, pomada, analgésico, antibióticos, não estavam dando resultado" (E2). Contudo, com a expansão da prática, tem havido um aumento no número de atendimentos 3 e conhecimento por parte dos pacientes e profissionais da saúde, passando a ser utilizada como tratamento complementar: "Ela mandou fazer acupuntura só para ajudar né, não que... o certo é fazer a cirurgia no pulso" (E5).

Uma terceira situação de encaminhamento identificada é por demanda do paciente: " $E u$ pedi, (...) porque há muitos anos atrás eu fiz acupuntura... eu tinha enxaqueca direto, desde criança... fiz acupuntura e nunca mais tive dor de cabeça" (E3).

Estudo realizado em Florianópolis mostrou que $88,7 \%$ da totalidade dos médicos e enfermeiros da saúde da família local desconheciam as diretrizes nacionais da PNPIC 23. Nesta pesquisa, 
Tabela 1

Características dos pacientes entrevistados.

\begin{tabular}{|c|c|c|c|c|c|c|}
\hline Entrevistado & Unidade & Sexo & $\begin{array}{l}\text { Idade } \\
\text { (anos) }\end{array}$ & $\begin{array}{c}\text { Escolaridade } \\
\text { (grau)* }\end{array}$ & $\begin{array}{l}\text { Renda mensal } \\
\text { familiar (R\$) }\end{array}$ & $\begin{array}{l}\text { Pessoas na } \\
\text { família ( } n \text { ) }\end{array}$ \\
\hline E1 & $A$ & $\mathrm{~F}$ & 55 & 1으 & $1.500,00$ & 4 \\
\hline E2 & & $M$ & 35 & 2으 & $2.500,00$ & 3 \\
\hline E3 & & $\mathrm{F}$ & 39 & 3o incompleto & $1.000,00$ & 3 \\
\hline E4 & & $\mathrm{F}$ & 53 & 1 으 & $1.300,00$ & 2 \\
\hline E5 & & $\mathrm{F}$ & 55 & 20 & $4.000,00$ & 4 \\
\hline E6 & B & $\mathrm{F}$ & 55 & 1 으 & $1.900,00$ & 2 \\
\hline E7 & & $\mathrm{F}$ & 48 & 2oㅇ & $2.500,00$ & 2 \\
\hline E8 & & M & 69 & 2으 & $3.500,00$ & 2 \\
\hline E9 & C & $\mathrm{F}$ & 43 & 2o & 700,00 & 9 \\
\hline E10 & & M & 86 & 1 으 & $2.500,00$ & 2 \\
\hline E11 & & $\mathrm{F}$ & 37 & 2oㅇ & $2.500,00$ & 2 \\
\hline E12 & & $\mathrm{F}$ & 34 & 20 & $1.500,00$ & 2 \\
\hline E13 & & $\mathrm{F}$ & 54 & 1 으 & $1.800,00$ & 2 \\
\hline E14 & & M & 65 & 1으 & $2.000,00$ & 3 \\
\hline E15 & & $\mathrm{F}$ & 54 & 3o & $3.100,00$ & 2 \\
\hline E16 & $D$ & M & 36 & 2o & $1.100,00$ & 2 \\
\hline E17 & & $\mathrm{F}$ & 70 & 3으 & $2.600,00$ & 4 \\
\hline E18 & B & $M$ & 50 & 2으 & $4.500,00$ & 4 \\
\hline E19 & & $\mathrm{F}$ & 40 & 1으 & 800,00 & 2 \\
\hline E20 & $E$ & $\mathrm{~F}$ & 57 & $1 \underline{0}$ & 600,00 & 2 \\
\hline E21 & & $\mathrm{F}$ & 58 & 20 & $2.500,00$ & 6 \\
\hline E22 & & $\mathrm{F}$ & 61 & 1 으 & $2.000,00$ & 2 \\
\hline E23 & $\mathrm{F}$ & $\mathrm{F}$ & 59 & 3으 & $1.500,00$ & 1 \\
\hline E24 & & $\mathrm{F}$ & 63 & 1 으 & 700,00 & 1 \\
\hline E25 & & $\mathrm{F}$ & 65 & 2oㅇ & $2.000,00$ & 2 \\
\hline E26 & $E$ & M & 46 & $1 \underline{0}$ & $1.100,00$ & 3 \\
\hline E27 & & $\mathrm{F}$ & 53 & 1ㅇ incompleto & $1.200,00$ & 3 \\
\hline E28 & $\mathrm{F}$ & M & 55 & 3으 & $3.000,00$ & 1 \\
\hline E29 & $D$ & $\mathrm{~F}$ & 39 & 1으 & $1.550,00$ & 8 \\
\hline E30 & & $\mathrm{F}$ & 41 & 2으 & $3.000,00$ & 2 \\
\hline
\end{tabular}

F: feminino; M: masculino.

* Por conveniência de formatação do quadro: 1o grau - Ensino Fundamental; 2o grau - Ensino Médio; 3o grau - Ensino Superior.

cerca de 30\% dos 177 entrevistados desconheciam a acupuntura, $50 \%$ tinham algum conhecimento e apenas $20 \%$ referiam ter maior entendimento sobre a técnica. Esse desconhecimento relativo pode ser devido à formação acadêmica que não incentiva e/ou pouco oferece disciplinas de outras racionalidades médicas, o que influencia a conduta profissional posterior 24. Em revisão sistemática, Otani \& Barros 12 identificaram vários autores discutindo a necessidade da implementação das MAC nas escolas médicas. Chistensen \& Barros 25 encontraram diferentes estudos mostrando o desejo de estudantes de medicina, de diversos países, de aprender sobre as MAC para usá-las com segurança e como ferramentas para a clínica.

O acesso dos pacientes na atenção primária à saúde, na maioria das vezes, se dá em um prazo menor em relação aos pacientes da atenção secundária. Pelo número limitado de atendimentos a dez sessões, somado à gravidade dos quadros, alguns pacientes da atenção secundária acabam frustrados, pois serão assistidos por um espaço de tempo por vezes insuficiente para a melhora esperada: "Se eu me sentir melhor, tô me sentindo emocionalmente melhor, mais disposta, dormindo melhor (...). Quer dizer, você começa o tratamento e é interrompido (...) eu acho que deveria 
ser mais nesses casos, de precisar mesmo" (E1). Muitos pacientes da atenção secundária aguardam anos na fila de espera: "Eu demorei dois anos para conseguir marcar” (E3).

$\mathrm{Na}$ atenção primária à saúde o atendimento geralmente já é sugerido pelo próprio médico que ali trabalha: "Foi sugestão do doutor mesmo, porque eu estava com uma dor no braço” (E8). Essas situações de demora nas filas e tratamento por um período limitado na atenção secundária, em que uma parte considerável da clientela parece chegar tardiamente à acupuntura, parecem corroborar a proposta de uma maior difusão desta prática na atenção primária à saúde, como direciona a PNPIC. Na atenção primária à saúde, a acupuntura, quando presente, se torna mais um recurso terapêutico dos profissionais, transformando-os, como denominou Barros 15, em profissionais híbridos, que podem escolher qual o melhor caminho terapêutico em cada momento 26 .

\section{Eficácia geral e uso de medicamentos na percepção dos usuários}

Dentre os entrevistados, a maioria relatou sentir melhora com o tratamento: "Setenta e cinco por cento do tamanho das lesões fecharam... eu fiquei maravilhada só pelo fato de diminuir a dor na minha perna" (E9). "Eu estou me sentindo melhor assim no caso da dor, tá aliviando, bastante (...). Eu não conseguia erguer para vestir uma blusa, agora está muito melhor (...). Agora consigo fazer tudo direito (no trabalho), antes sempre fazia com cuidado por causa do braço" (E12). Alguns poucos não observaram benefícios: "Então como é a oitava sessão que eu tô fazendo, não sei, acho que não vai resolver meu problema, não vai me tirar as dores, entendeu?" (E1). A maior parte dos pacientes que não relataram melhoras significativas era da atenção secundária (cinco) e apresentavam doenças com maior gravidade; dois eram da atenção primária à saúde.

Notou-se um grande número de encaminhamentos por quadros de dor, que, segundo os profissionais acupunturistas, é o mais frequente motivo de uso da acupuntura, cerca de $80 \%$ dos pacientes. Os entrevistados mensuraram a melhora, nos casos de quadros dolorosos, em relação ao início do tratamento: dos 27 com quadros álgicos, 19 relataram melhora de $60 \%$ a $100 \%$ na dor, três melhoraram entre $30 \%$ e $50 \%$, três de $10 \%$ a $20 \%$, um não sentiu melhora e um não soube avaliar. Esse resultado positivo converge com os abundantes estudos sobre a eficácia dessa terapêutica 1,27,28. Esse efeito é bem-vindo nos casos de dor, porém, deixa intacta a questão da dependência do procedimento e dos significados envolvidos no problema, bem como a questão do autocuidado. Nesse sentido, a acupuntura, em tese, pode ser apenas um procedimento complementar ou em alguns casos substitutivo aos fármacos, sem alterar o quadro geral das representações dos pacientes e sua participação no cuidado. Ao contrário, pode exigir maior deslocamento e tempo gasto, já que demanda a aplicação heterônoma das agulhas, diferentemente da medicação que pode ser administrada em casa.

Vários usuários apresentavam problemas crônicos com longo itinerário de tratamento farmacológico e/ou cirúrgico sem, muitas vezes, conseguirem melhora. No caso das dores crônicas, utilizavam com frequência anti-inflamatórios, cujo uso contínuo pode provocar reações adversas relativamente frequentes e bem conhecidas 29,30,31. Observou-se casos de iatrogenias medicamentosas: "Eu tomei vários remédios, hoje em dia eu tenho gastrite, não é todo remédio que eu posso tomar" (E1).

Os avanços terapêuticos ocorridos nas últimas décadas são inquestionáveis, no entanto, os medicamentos sempre foram venenos potenciais e seus efeitos não desejados aumentaram com a eficácia e a extensão de seu uso ${ }^{10}$. As indústrias farmacêuticas lançam, a cada dia, novos medicamentos ${ }^{32}$. Essa indústria, segundo Conrad 9 , tem exercido um importante papel no processo de medicalização social. Ela atua na mídia apresentando artistas famosos, atletas, etc. em suas propagandas, na tentativa de exibir símbolos de status, poder, sucesso, força, beleza e juventude 33. O mercado farmacêutico está sempre lançando novas marcas e influencia a população a consumir os medicamentos ditos "mais modernos". Os médicos muitas vezes se veem pressionados a prescrever o medicamento mais novo, com receio de se sentirem obsoletos 34 .

Segundo Menéndez 35, a biomedicina segue depositando suas expectativas em investigações que geram explicações biológicas para os padecimentos e soluções baseadas em fármacos específicos, alimentando a biologização das representações sociais do processo saúde/ enfermidade/atenção. Os fármacos podem gerar, principalmente em longo prazo, uma série de prejuízos. Apesar dos limites da acupuntura, esta pode ser um tratamento alternativo ou coadjuvante, propiciando redução do uso de quimioterápicos. Isso foi observado em alguns relatos: “Medicação, vou ser sincero, não tomei mais, depois da acupuntura eu parei de tomar"(E2). "Durante um ano fazendo acupuntura, consegui ficar três meses sem tomar morfina e o tempo que tomava não foi na mesma intensidade" (E9). "Eu vivia dopada, direto. Agora raramente eu tomo um anti-inflamatório" (E19). 
Devido aos efeitos adversos de curto e longo prazos causados pelo tratamento medicamentoso e os riscos de dependência, principalmente em patologias crônicas, nossos achados e outros estudos da literatura 31 indicam que a acupuntura pode contribuir para diminuir a polimedicação e as iatrogenias medicamentosas, além de melhorar a potência do cuidado. Kooreman \& Baars 36 mostraram não só menores custos com o uso de outras racionalidades médicas (acupuntura, homeopatia e medicina antroposófica) em pacientes da atenção primária à saúde europeia, como também maior longevidade dos mesmos.

\section{Melhora de outros aspectos além do problema principal}

As dores físicas costumam ser a porta de entrada para o atendimento em acupuntura. No entanto, é comum e desejável que sejam levados em consideração pelo acupunturista (e demais profissionais) outros desequilíbrios ou queixas, aspectos subjetivos e a história individual de cada um. A MTC propicia interpretações dos adoecimentos baseada em um pensamento no qual os fenômenos são compreendidos como eventos que estão conectados entre si 19,26. Os sinais e os sintomas do indivíduo, o meio em que vive, suas emoções, relações pessoais, etc., são relevantes para o diagnóstico e o quadro clínico é visualizado como um todo, podendo a acupuntura propiciar benefícios em múltiplos problemas simultaneamente 37 . Nesse sentido, investigou-se se os usuários sentiam melhoras também em outros aspectos além do problema principal, motivo de sua procura pelo serviço e/ou terapêutica.

Dentre os 13 entrevistados da atenção primária à saúde, 11 relataram sentir melhoras em outros aspectos além da queixa principal e 10 dos 17 pacientes da atenção secundária, enquanto que dois da atenção primária à saúde e sete da atenção secundária não sentiram. Alguns relatos qualificam esses achados: "É eu melhorei mais, assim no humor eu tô assim mais disposta agora (...) aquilo ali que eu não fazia, de repente eu vô lá e faço, entendeu? (...) De repente até minha autoestima. Fui arrumar o cabelo, coisa que já fazia muito tempo que eu não ligava mais para isso (E1)". "Pra depressão um pouco acho que tem ajudado sim, porque eu tinha muita angústia, muita assim, todos os dias, hoje já tá mais raras assim, não é todo o dia como eu tinha. No sono, parece que tá mais tranquilo, que eu acordava às vezes à noite e agora eu tô dormindo assim mais direto" (E4).

Segundo Alves 38, a integralidade, um dos princípios do SUS, contrapõe-se à abordagem fragmentária e reducionista do indivíduo. O olhar do profissional, nesse sentido, deve ser totalizante, buscando apreender as necessidades mais abrangentes dos sujeitos. Embora a biomedicina reconheça a existência da dor "psicossomática” ou "psicogênica”, sua atitude para com a dor tende a não levar em conta elementos sociais, culturais, morais e psicológicos que estão associados muitas vezes 14. A biomedicina apresenta tendências de objetivação dos problemas e dos usuários, e tem sido questionada pelas vivências de desarmonia na relação médico/paciente, bem como pelas suas iatrogenias clínicas. As abordagens mais holísticas das MAC se destacam pela sua diferente forma de lidar com o paciente, ao levar em conta aspectos mais sutis e subjetivos da vida que não se restringem apenas à patologia em si 24,39. A acupuntura, juntamente com outras MAC, pode ser uma opção nesse sentido. Os relatos dos pacientes revelam uma abordagem que abrange outros problemas além do principal. Nesse sentido, ela mostra uma abrangência significativa, conforme conceituação de Starfield 40, aproximandose, ainda que de forma limitada, da ideia de integralidade, de humanização do cuidado e de clínica ampliada 40,41 .

No Brasil, convergente com essa ideia, destaca-se a Política Nacional de Humanização (PNH), elaborada em 2004, configurada em torno da humanização das práticas de atenção e gestão no sistema de saúde. Dentre os princípios norteadores da $\mathrm{PNH}$, estão: o fomento da autonomia e do protagonismo dos pacientes e o aumento do grau de corresponsabilidade na produção de saúde entre usuários e profissionais de saúde, e valorização da dimensão subjetiva e social em todas as práticas de atenção 42 . O caráter mais holístico e integral da abordagem das racionalidades médicas vitalistas e sua visão positiva de saúde podem ter efeitos colaterais medicalizantes, como salientou Camargo Jr. ${ }^{43}$. Segundo o autor, sua grande "sensibilidade" e seu caráter inclusivo e ampliado podem gerar medicalização de sintomas e aspectos diversos das pessoas. Todavia, nossos resultados indicam que isso pouco ou não ocorreu com os entrevistados. Neles, a melhora de outros sintomas acompanhou o tratamento dos problemas principais. Apesar de o acesso à acupuntura ser restrito no SUS, tanto na atenção primária à saúde como na atenção secundária, devido ao pequeno número de profissionais praticantes, e isto tenha possivelmente um efeito seletivo sobre os tipos de demandas atendidas, somente com a maior oferta desse cuidado talvez se possa perceber e investigar se a "armadilha" medicalizante aventada pelo autor é significativa no caso da acupuntura. 


\section{Modelos explicativos e autocuidado}

Investigou-se a influência da acupuntura sobre o autocuidado e o modelo explicativo dos usuários, questão central na discussão da medicalização do cuidado. As concepções e representações sociais dos pacientes são formadas com base na sua experiência de vida no seu meio sociocultural, e comumente os usuários de alguma forma as reconstroem conforme seu contato e experiência com os vários tipos de cuidado, conhecimento e tratamento com os quais interagem ${ }^{44}$, na busca da resolução de seus problemas de saúde. Podese usar o conceito de Kleinman 45 , de modelos explicativos, que consiste em explicações de um episódio de doença e do cuidado terapêutico, revelados mediante as narrativas dos sujeitos 14,45. As explicações e entendimentos dos pacientes sobre seus problemas (seu modelo explicativo) vão exprimir suas concepções prévias, de alguma forma modificadas ou influenciadas pelos saberes e tecnologias experimentados no cuidado recebido e no autocuidado.

As representações sociais aí envolvidas podem ser mais ou menos medicalizadas 39 , tanto pela própria cultura do doente quanto pelo seu comum prévio contato com a biomedicina. A experiência com a acupuntura é processada de alguma forma, alimentando mais ou menos sua autonomia relativa, modificando (ou não) suas representações sociais e seu modelo explicativo, podendo se dar em vários sentidos, mais ou menos medicalizantes.

Notou-se que, para a maioria, a intervenção da acupuntura e seus significados se misturavam com conceitos e noções biomédicas, no sentido de não se aproximarem do entendimento de outra racionalidade médica: "Artrose é uma doença degenerativa e que não tem cura, quer dizer este tratamento é paliativo, mas me deu uma qualidade de vida muito boa" (E10). "Éos pontos que pega manda para o cérebro da gente e o cérebro que manda aquela dosagem da medicação né, daí faz um monte de medicação que o cérebro envia para onde está inflamado, para as dores" (E6).

Apesar de muitos se sentirem bem com o tratamento com acupuntura, não incorporaram aprendizados ou noções oriundas da MTC. Confiavam em seus terapeutas e pouco questionavam sobre a técnica: "Pelo jeito é coisa boa, que se fosse coisa ruim ninguém ia fazer (...) Eu me sinto bem, meu organismo aceitou bem isso, meu corpo também aceitou, então, isso é o que importa pra mim" (E28). Com efeito, tal achado sincrético é esperado em boa medida, pois normalmente o doente valoriza mais a relação com o terapeuta e o resultado empírico. Isso por si já o deixa tranquilo e confiante, sem despertar a necessidade de entender todo o processo de tratamento 46. Pode-se considerar previsível encontrar uma compreensão superficial e leiga do problema pelos usuários, em comparação com um ponto de vista especializado, seja biomédico, seja da MTC 47 . Todavia, a raridade de menção a outras noções, entendimentos ou interpretações dos seus problemas faz pensar que esses profissionais utilizam a acupuntura, no contato com os doentes, de forma um tanto integrada e ou subordinada ao paradigma biomédico. E ainda (ou ainda), se usam a MTC como referencial na sua abordagem e no seu "raciocínio clínico", pouco se comunicam com seus usuários sobre isso perdendo, assim, algo de suas potencialidades.

O lado educativo do tratamento, ou da relação profissional/doente, é envolvido no processo do cuidado e é particularmente importante nos casos crônicos. Sabe-se que a tradição pedagógica biomédica tende a se caracterizar por uma atitude autoritária, monologal, diretiva e pouco sensível às realidades psicossociais dos doentes e seus modelos explicativos 38,39,48. Foram investigadas as recomendações realizadas pelos terapeutas durante o tratamento. Analisou-se se esses incentivavam práticas de autocuidado e como os saberes e as várias formas de cuidado da MTC se faziam presentes para os pacientes, contribuindo para a melhor elaboração dos sofrimentos ou pluralizando os sentidos dos adoecimentos.

Em muitos casos, principalmente vistos na atenção secundária, as orientações não diferiram das utilizadas na prática biomédica: "Se tiver dor fazer compressa com água morna (...). Fazer alongamento, (...) os exercícios que eles me deram na fisioterapia" (E11).

No entanto, alguns casos foram observados na atenção primária à saúde em que outras orientações, não biomédicas ou ao menos não consagradas ou comuns nessa racionalidade eram exploradas, possivelmente relacionadas à MTC: "Eu aprendi a respirar também (...) Respiro tranquila, faço massagem em casa para dormir e ele sempre tá dando uma dica ou outra (...) e eu procuro fazer tudo né... cuido da alimentação. $O$ doutor tá me ensinando muita coisa disso também” (E7). "Quando eu sinto alguma coisa, eu faço, tenho aquele manual dele lá [massagem], dos pontos... Eu fiz para dor de cabeça, eu sou meio esquecido, dai tenho que ir no manual para ver os pontinhos (...) vai fazendo né, se dói a cabeça vai aqui, vai lá no pé, no dedão" (E8).

Os usuários da atenção primária à saúde são atendidos por profissionais que os acompanham há mais tempo, conhecem o meio em que vivem e possuem com eles maior familiaridade, podendo contribuir com orientações mais voltadas à sua realidade. Já a prática na atenção secundária 
mostrou-se mais engessada, tendendo a tratar a queixa principal e restringindo-se, administrativamente, a um número limitado de dez sessões que, em muitos casos, não é o suficiente para formar um vínculo mais estreito entre terapeuta e paciente.

Por outro lado, o pacote de dez sessões pode ser um tempo necessário para atenuar os sintomas das queixas apresentadas e encaminhar para outros recursos que incentivem o autocuidado, não deixando o usuário dependente apenas da acupuntura. Notou-se em alguns atendimentos as orientações de uns médicos para que seus pacientes iniciassem práticas corporais: "Mandou que eu procurasse fazer alongamentos (...) até agora recentemente sugeriu que eu fizesse yoga, que tem um professor fazendo em (...) e que iria me ajudar bastante" (E21). De acordo com Luz 49, a acupuntura pode despertar certa "passividade" no paciente e essas práticas corporais se destacam por propiciar um maior conhecimento de si mesmo, de seu corpo e de seu psiquismo. Porém, a existência de oferta de outras práticas da MTC não ocorria nos serviços municipais como regra geral, os quais, na época da coleta de dados, não dispunham de apoio institucional para a prática das MAC.

Destacou-se, dentre os profissionais da atenção primária à saúde, um que tinha a iniciativa de oferecer aos seus pacientes outros recursos da MTC, viabilizados por meio de um encontro coletivo para conversa, orientação sobre a MTC e o ensino de pontos para automassagem, inclusive com fornecimento de material educativo (relatado na citação de E8). Esse profissional foi um dos poucos mencionados como enriquecedor dos modelos explicativos (com possibilidades interpretativas da MTC) e dos cuidados por meio de outras práticas da MTC. Isso remete à questão da atitude e dos conhecimentos dos acupuntores. Esses podem exercitar de distintas formas seu cuidado e sua relação com os usuários, o que traz à discussão sua formação e sua competência. Essa última pode ser restrita à técnica da acupuntura, o que talvez seja o caso da maior parte dos acupunturistas médicos brasileiros (todos os acupunturistas dos entrevistados eram médicos). A formação dos acupunturistas no SUS (na atenção secundária e na atenção primária à saúde) pode reforçar o relativo isolamento desta terapêutica do conjunto dos saberes e práticas da MTC. Tal isolamento já está em curso com a institucionalização da acupuntura na biomedicina e relaciona-se, provavelmente, ao maior ou menor caráter (des)medicalizante da mesma.

Todavia, a formação e competência dos profissionais não foram concebidas como objetos deste estudo e este é um limite do mesmo, que sugere a necessidade de outras pesquisas. Outra limitação é que o mesmo foi realizado em apenas seis serviços de um único município, inviabilizando generalizações para outros contextos. Por último, a abordagem do tema, via entrevistas, apresenta o limite, quanto à questão investigada, de não enfocar diretamente o processo de cuidado com a acupuntura propriamente dita, e sim captar a versão relatada pelo usuário que o estava vivenciando naquele momento, na medida da possibilidade do diálogo que uma única entrevista permitia no ambiente do serviço de saúde.

\section{Considerações finais}

A maioria dos pacientes chegou à acupuntura na atenção secundária quando outros tratamentos falharam e em estágios avançados de adoecimento. Mesmo nesses casos, ela foi percebida pela maioria dos usuários com relativa eficácia sob vários aspectos, com melhoria de seus sofrimentos e quadros álgicos e redução do uso de medicamentos. Esse é um ponto importante, devendo ser mais bem estudada a hipótese da utilização da acupuntura significar efetivamente redução de uso, danos e custos com medicamentos.

Apesar da dificuldade de acesso, já que poucos profissionais da atenção primária à saúde são acupunturistas praticantes, foi observado que estes possuem uma maior autonomia para atender aos pacientes, podendo tratar por mais tempo casos mais graves. O ambiente da atenção primária à saúde parece favorecer o uso da acupuntura e de outras modalidades da MTC. O acesso à acupuntura é precário e deve ser visto com cuidado pelos gestores, já que a lista de espera na atenção secundária tende a ser grande e lenta e os profissionais capacitados na atenção primária à saúde são poucos, com pouco tempo disponível e dificuldades para a prática.

Os resultados mostraram que a acupuntura pouco tem contribuído para a autonomia e desmedicalização, salvo pela sua efetividade. Ela pode, sob a ótica do paciente, ser confundida como apenas mais uma especialidade ou terapêutica biomédica, como observado na maioria dos casos neste estudo, sem contribuir para a ampliação dos significados dos adoecimentos, das modalidades de cuidado e do empoderamento dos doentes. Nesse sentido, a atitude do profissional é importante para estimular uma postura mais ativa e integrante. A acupuntura facilita essa postura por sua abordagem vitalista e de cuidado ampliado, possibilitando, mas não garantindo, que os terapeutas estimulem o autocuidado. A oferta pelo SUS, tanto na atenção primária à saú- 
de quanto na atenção secundária, de outras modalidades de cuidado e de promoção da saúde pela MTC certamente facilita o estímulo ao seu uso pelos pacientes e sua recomendação pelos demais profissionais, valorizando a cosmovisão e as variadas práticas desta medicina 19 .

Quando considerada apenas como um recurso para tratamento de problemas clínicos isolados, a acupuntura mostrou ter um caráter relativamente menos medicalizante se comparada com os tratamentos biomédicos convencionais, sobretudo nos casos de dor crônica, para o que já é muito usada, funcionando como uma via de "redução de danos" em relação à biomedicina 50 .

Novas pesquisas enfocando os processos de realização do cuidado com acupuntura e MTC são necessárias para a melhor compreensão e análise de sua atuação, em termos de potencialidades e limitações no SUS, na atenção primária à saúde e nos demais serviços da rede de atenção.

\section{Resumen}

Las virtudes atribuidas a las terapias complementarias con enfoque holístico, centradas en los pacientes y que estimulan la auto-curación, han sido valoradas y pueden, en teoría, aliviar la excesiva medicalización social. Entre ellas, la acupuntura se distingue por la institucionalización progresiva y su aceptación. El objetivo fue analizar la experiencia de los pacientes con tratamientos de acupuntura en el Sistema Nacional de Salud y cómo se ajusta a sus condiciones, así como la visión, representaciones y modelos explicativos de los usuarios respecto a la medicalización social. Entrevistamos a 30 pacientes de atención primaria y secundaria de salud de Florianópolis, Santa Catarina, Brasil. Observamos los resultados en las principales quejas, el sueño, el estado de ánimo, el estado emocional y la disminución del consumo de drogas. Los modelos explicativos de los usuarios y de cuidado personal eran pocos o no modificados por el tratamiento. Se observó, sin embargo, una amplitud de la atención y se consideró menos iatrogénica, principalmente en la atención primaria de salud, en la que había poca oferta de otras prácticas de medicina china.

Acupuntura; Terapias Complementarias; Autocuidado; Atención Primaria de Salud

\section{Colaboradores}

E. D. C. Silva e C. D. Tesser participaram conjuntamente de todas as fases da pesquisa e da elaboração do artigo: concepção e planejamento da pesquisa, coleta e análise dos dados, revisões bibliográficas, redação inicial, revisões e redação final do artigo. Na coleta a participação de C. D. Tesser restringiu-se a poucas entrevistas iniciais.

\section{Agradecimentos}

Aos profissionais acupunturistas pela receptividade durante a coleta de dados e aos pacientes que se prontificaram em fazer parte desta pesquisa. 


\section{Referências}

1. World Health Organization. Acupuncture: review and analysis of reports and controlled clinical trials. Geneva: World Health Organization; 2002.

2. Luz MT. Medicina e racionalidades médicas: estudo comparativo da medicina ocidental contemporânea, homeopática, chinesa e ayurvédica. In: Canesqui AM, organizadora. Ciências sociais e saúde para o ensino médico. São Paulo: Editora Hucitec; 2000. p. 181-200.

3. Sousa IMC, Bodstein RCA, Tesser CD, Santos FAZ, Hortale VA. Práticas integrativas e complementares: oferta e produção de atendimentos no SUS e em municípios selecionados. Cad Saúde Pública 2012; 28:2143-54.

4. Ministério da Saúde. Portaria no 971/06. Dispõe sobre a integralidade da atenção como diretriz do SUS e aprova a Política Nacional de Práticas Integrativas e Complementares (PNPIC) no Sistema Único de Saúde. Diário Oficial da União 2006; 3 mai.

5. Santos FAZ, Gouveia GC, Martelli PJL, Vasconcelos EMR. Acupuntura no Sistema Único de Saúde e a inserção de profissionais não-médicos. Rev Bras Fisioter 2009; 13:330-4

6. Freire Jr. MB. Conhece-te a ti mesmo: uma proposta de educação popular para a saúde. Saúde Debate 1993; (41):4-8.

7. Cintra MER, Figueiredo R. Acupuntura e promoção de saúde: possibilidades no serviço público de saúde. Interface Comun Saúde Educ 2010; 14:13954.

8. Vas J, Vega E, Méndez C. Tratamiento con acupuntura del dolor crónico benigno. Medicina de Familia (Andalucía) 2002; 4:239-44.

9. Conrad P. The medicalization of society: on the transformation of human conditions into treatable disorders. Baltimore: The Johns Hopkins University Press; 2007.

10. Illich I. A expropriação da saúde: nêmesis da medicina. Rio de Janeiro: Editora Nova Fronteira; 1975.

11. Tesser CD. Medicalização social (II): limites biomédicos e propostas para a clínica na atenção básica. Interface Comun Saúde Educ 2006; 10:347-62.

12. Otani MAP, Barros NF. A medicina integrativa e a construção de um novo modelo de saúde. Ciênc Saúde Coletiva 2011; 16:1801-11.

13. Carvalho SR, Gastaldo D. Promoção à saúde e empoderamento: uma reflexão a partir das perspectivas crítico-social pós-estruturalista. Ciênc Saúde Coletiva 2008; 13:2029-40.

14. Helman CG. Cultura, saúde e doença. 5ạ Ed. Porto Alegre: Editora Artmed; 2009.

15. Barros NF. A construção da medicina integrativa: um desafio para o campo da saúde. São Paulo: Editora Hucitec; 2008.

16. Luz MT, Barros NF, organizadores. Racionalidades médicas e práticas integrativas em saúde: estudos teóricos e empíricos. Rio de Janeiro: Instituto de Medicina Social, Universidade do Estado do Rio de Janeiro/ABRASCO; 2012. (Coleção Clássicos para Integralidade em Saúde).
17. Tesser CD. Práticas complementares, racionalidades médicas e promoção da saúde: contribuições poucos exploradas. Cad Saúde Pública 2009; 25:1732-42.

18. Sousa IMC, Vieira ALS. Serviços públicos de saúde e medicina alternativa. Ciênc Saúde Coletiva 2005; 10 Suppl:255-66.

19. Palmeira GA. A acupuntura no ocidente. Cad Saúde Pública 1990; 6:117-28.

20. Minayo MCS. O desafio do conhecimento: pesquisa qualitativa em saúde. 2a Ed. Rio de Janeiro: ABRASCO/São Paulo: Editora Hucitec; 1999.

21. Fontanella BJB, Ricas J, Turato ER. Amostragem por saturação em pesquisas qualitativas em saúde: contribuições teóricas. Cad Saúde Pública 2008; 24:17-27.

22. Bardin L. Análise de conteúdo. Lisboa: Edições 70; 1979.

23. Thiago SCS, Tesser CD. Percepção de médicos e enfermeiros da Estratégia de Saúde da Família sobre terapias complementares. Rev Saúde Pública 2011; 45:249-57.

24. Queiroz MS. O itinerário rumo às medicinas alternativas: uma análise em representações sociais de profissionais da saúde. Cad Saúde Pública 2000; 16:363-75.

25. Christensen MC, Barros NF. Medicinas alternativas e complementares no ensino médico: revisão sistemática. Rev Bras Educ Méd 2009; 34:97-105.

26. Cunha GT. A construção da clínica ampliada na atenção básica. São Paulo: Editora Hucitec; 2005.

27. Koo ST, Park YI, Lim KS, Chung K, Chung JM. Acupuncture analgesia in a new rat model of ankle sprain pain. Pain 2002; 99:423-31.

28. Lundeberg T, Stener-Victorin E. Is there a physiological basis for the use of acupuncture in pain? International Congress Series 2002; 1238:3-10.

29. Goodman LS, Gilman A. As bases farmacológicas da terapêutica. Rio de Janeiro: McGraw Hill; 1996.

30. Süsskind M, Thürmann PA, Lüke C, Jeschke E, Tabali $\mathrm{M}$, Matthes $\mathrm{H}$, et al. Adverse drug reactions in a complementary medicine hospital: a prospective, intensified surveillance study. Evid Based Complement Alternat Med 2012; 2012:320760.

31. Meng CF, Wang D, Ngeow J, Lao L, Peterson M, Paget S. Acupuncture for chronic low back pain in older patients: a randomized, controlled trial. Rheumatology (Oxford) 2003; 42:1508-17.

32. Dupuy JP, Karsenty S. A invasão farmacêutica. Rio de Janeiro: Edições Graal; 1979.

33. Nascimento MC. Medicamentos, comunicação e cultura. Ciênc Saúde Coletiva 2005; 10:179-93.

34. Camargo Jr. K. Medicalização: um quadro de referência. In: Pinheiro R, Silva Jr. AG, organizadores. Por uma sociedade cuidadora. Rio de Janeiro: Centro de Estudos e Pesquisa em Saúde Coletiva, Instituto de Medicina Social, Universidade do Estado do Rio de Janeiro/ABRASCO; 2010. p. 209-16. 
35. Menéndez EL. Sujeitos, saberes e estruturas: uma introdução ao enfoque relacional no estudo da saúde coletiva. São Paulo: Aderaldo \& Rothschild; 2009.

36. Kooreman P, Baars EW. Patients whose GP knows complementary medicine tend to have lower costs and live longer. Eur J Health Econ 2012; 13:769-76.

37. Sumoto T, Terasawa S. Influence of acupuncture and moxibustion on QOL of the elderly living in nursing home and care house. Nihon Ronen Igakkai Zasshi 2001; 38:205-11.

38. Alves VS. Um modelo de educação em saúde para o Programa Saúde da Família: pela integralidade da atenção e reorientação do modelo assistencial. Interface Comun Saúde Educ 2005; 9:39-52.

39. Tesser CD, organizador. Medicalização social e atenção à saúde no SUS. São Paulo: Editora Hucitec; 2010.

40. Starfield B. Atenção primária: equilíbrio entre necessidades de saúde, serviços e tecnologia. Brasília: Organização das Nações Unidas para a Educação, a Ciência e a Cultura/Ministério da Saúde; 2002.

41. Ministério da Saúde. Política Nacional de Humanização da Atenção e Gestão do SUS: clínica ampliada e compartilhada. Brasília: Ministério da Saúde; 2009.

42. Ministério da Saúde. HumanizaSUS: Política Nacional de Humanização. A humanização como eixo norteador das práticas de atenção e gestão em todas as instâncias do SUS. Brasília: Ministério da Saúde; 2004.

43. Camargo Jr. KR. As armadilhas da "concepção positiva de saúde”. Physis (Rio J.) 2007; 17:63-76.

44. Tesser CD. Racionalidades médicas e integralidade: desafios para a saúde coletiva e o SUS. In: Pinheiro R, Silva Jr. AG, organizadores. Por uma sociedade cuidadora. Rio de Janeiro: Centro de Estudos e Pesquisa em Saúde Coletiva, Instituto de Medicina Social, Universidade do Estado do Rio de Janeiro/ABRASCO; 2010. p. 77-100.
45. Kleinman A. Orientations 3: core clinical functions and explanatory models. In: Kleinman A, editor. Patients and healers in the context of culture: an exploration of the borderland between anthropology, medicine, and psychiatry. Berkeley: University of California Press; 1980. p. 71-118.

46. Tesser CD, Luz MT. Racionalidades médicas e integralidade. Ciênc Saúde Coletiva 2008; 13:195-206.

47. Bellotto Jr. N, Martins LC, Akerman M. Impacto dos resultados no tratamento por acupuntura: conhecimento, perfil do usuário e implicações para promoção da saúde. Arq Méd ABC 2005; 30:83-6.

48. Machado MFAS, Monteiro EMLM, Queiroz DT, Vieira NFC, Barroso MGT. Integralidade, formação de saúde, educação em saúde e as propostas do SUS: uma revisão conceitual. Ciênc Saúde Coletiva 2007; 12:335-42.

49. Luz MT. Cultura contemporânea e medicinas alternativas: novos paradigmas em saúde no século XX. Physis (Rio J.) 2005; 15 Suppl:145-76.

50. Tesser CD, Barros NF. Medicalização social e medicina alternativa e complementar: pluralização terapêutica do Sistema Único de Saúde. Rev Saúde Pública 2008; 42:914-20.

Recebido em 31/Out/2012

Versão final reapresentada em 24/Mar/2013

Aprovado em 03/Jun/2013 